\title{
Light sensitive memristor with bi-directional and wavelength-dependent conductance control
}

\author{
P. Maier, ${ }^{1}$ F. Hartmann, ${ }^{1, a)}$ M. Rebello Sousa Dias, ${ }^{2,3}$ M. Emmerling, ${ }^{1}$ \\ C. Schneider,${ }^{1}$ L. K. Castelano, ${ }^{2}$ M. Kamp,${ }^{1}$ G. E. Marques,${ }^{2}$ V. Lopez-Richard,${ }^{2}$ \\ L. Worschech ${ }^{1}$ and S. Höfling ${ }^{1,4}$ \\ ${ }^{1}$ Technische Physik and Wilhelm Conrad Röntgen Research Center for Complex Material Systems, \\ Physikalisches Institut, Universität Würzburg, Am Hubland, D-97074 Würzburg, Germany. \\ ${ }^{2}$ Departamento de Fisica, Universidade Federal de São Carlos, 13565-905 São Carlos, SP, Brazil. \\ ${ }^{3}$ present address: Institute for Research in Electronics and Applied Physics, University of Maryland, College \\ Park, Maryland 20742, United States. \\ ${ }^{4}$ SUPA, School of Physics and Astronomy, University of St. Andrews, St. Andrews, KY16 9SS, United \\ Kingdom.
}

We report the optical control of localized charge on positioned quantum dots in an electro-photo-sensitive memristor. Interband absorption processes in the quantum dot barrier matrix lead to photo-generated electron-hole-pairs that, depending on the applied bias voltage, charge or discharge the quantum dots and hence decrease or increase the conductance. Wavelength-dependent conductance control is observed by illumination with red and infrared light, which leads to charging via interband and discharging via intraband absorption, respectively. The presented memristor enables optical conductance control and may thus be considered for sensory applications in artificial neural networks as light-sensitive synapses or optically tunable memories.

\footnotetext{
${ }^{\text {a)} C o r r e s p o n d i n g ~ a u t h o r ; ~ e l e c t r o n i c ~ m a i l: ~ f a b i a n . h a r t m a n n @ p h y s i k . u n i-w u e r z b u r g . d e . ~}$
} 
Semiconductor quantum dots embedded in optoelectronic devices are appealing for applications such as lasers, ${ }^{1}$ single photon sources, ${ }^{2}$ spin memories $^{3}$ and floating gate transistors. ${ }^{4}$ Floating gate transistors are key building blocks of flash memories and store information by means of localized charge on a floating gate. ${ }^{5}$ Tuning the amount of charge with gate terminals allows to change the resistance of a nearby twodimensional electron gas (2-DEG). ${ }^{6}$ Realizing floating gates with quantum dots based on III-Vsemiconductors further provides optical and wavelength-selective control of the resistance, ${ }^{7-9}$ which can be exploited for single photon detection ${ }^{10}$ or optical memories. ${ }^{11,12}$ In addition, floating gate transistors can be used to realize a memristive operation mode. ${ }^{13,14}$ Memristors are passive fundamental circuit elements with a voltage controllable resistance ${ }^{15,16}$ and promising candidates for future computing architectures with inherent memory. ${ }^{17}$ The realization of optoelectronic memristors with optical readout, ${ }^{18-20}$ optoelectronic tuning of the resistance state ${ }^{21-23}$ and light-induced one-directional conductance change $^{24-26}$ allows to perform arithmetic operations..$^{25,27}$ Controlling memristors solely by light and realizing photonic synapses however requires bi-directional optical tuning of the resistance. ${ }^{28}$ In addition, wavelength-dependent conductance control is beneficial for sensory applications and enables optical communication of electronic devices by converting information of the wavelength to a resistance. ${ }^{25}$

We present bi-directional and wavelength-dependent conductance changes of a quantum dot memristor. The memristor is realized on the mature III-V-semiconductor material platform and the conductance corresponds to different amounts of localized electrons on positioned quantum dots (QDs). Illumination of the device with infrared and red light allows to increase and decrease the conductance via intraband and interband absorption, respectively.

The presented device is based on a modulation doped GaAs/AlGaAs heterostructure. A wire and lateral gates are defined via electron beam lithography and dry chemical etching (see Fig. 1(a)). QDs are 
positioned along the wire by growing InAs on predefined holes (details on fabrication in Ref. 29). The presented measurements are conducted at $4.2 \mathrm{~K}$. Memristive operation of the device is realized by connecting the drain contact of the wire with the lateral gates (as diode connected transistor, see Fig. 1(a)). ${ }^{30}$ Fig. 1(b) shows the current-voltage-characteristic of the device in the dark and under cw illumination with a red light emitting diode (LED). The photon energy of the LED is $2 \mathrm{eV}$ and above the band gap energies of the InAs QDs $(\approx 1.35 \mathrm{eV})$ and the surrounding GaAs $(\approx 1.50 \mathrm{eV})$ and AlGaAs $(\approx 1.75 \mathrm{eV})$ barriers. In the dark, a pinched hysteresis loop as finger print of memristive operation is evident. ${ }^{31}$ The pinched hysteresis loop is characterized by a high $(G=0.7 \mu \mathrm{S})$ and a low conductance state $\left(G=1.4 \times 10^{-5} \mu \mathrm{S}\right)$ around zero bias voltage. Under cw illumination with a light power below $1 \mathrm{nW}$ (detection limit of our photosensor), only the high conductance state is evident at zero bias. The high and low conductance states correspond to small and large amounts of localized electrons on the QDs, respectively, ${ }^{30}$ and hence, the QDs are completely discharged by the low power optical excitation. Within the voltage range from -1 to $-3 \mathrm{~V}$ a reduction of the conductance under illumination is observed. The lower conductance implies that the QDs can be optically charged as well. Thus, depending on the bias voltage, optically activated charging and discharging processes occur.

Fig. 1(c) shows the conductance of the device for pulsed optical excitation with pulse widths of $\Delta t=10 \mu \mathrm{s}$ and different light powers. During the application of the pulses, the bias voltage is set to $-1.8 \mathrm{~V}$. After every optical pulse a voltage pulse with amplitude $1.0 \mathrm{~V}$ is applied to determine the conductance. Prior to the measurement, the QDs are discharged and the conductance is maximum with $G \approx 0.7 \mu \mathrm{S}$. For a power of $3 \mathrm{nW}$, the conductance remains almost unchanged up to 2000 pulses. Larger light powers allow to successively lower the conductance with the onset occurring at smaller pulse numbers. Setting the bias voltage during illumination to zero, the conductance increases as depicted in Fig. 1(d). The light power is $44 \mu \mathrm{W}$ and the pulse width is varied. Prior to the measurement, the QDs are charged. For a width of 
$100 \mathrm{~ns}$, the conductance remains zero up to 2000 pulses. Larger widths lead to an increase of the conductance and less pulses are required for the onset. The increase and decrease of the conductances correspond to optical discharging and charging of the QDs, respectively. Because the retention time of the present device is longer compared to the timescale of the experiments, time dependent conductance changes are negligible for the presented experiments.

The bias voltage dependency of the charging and discharging processes is explained by the band profiles of the conduction (CB) and valence band (VB), as illustrated in Figs. 1(e) and (f). The photon energy is sufficient to create electron-hole-pairs in the GaAs/AlGaAs heterostructure around the QDs via interband absorption. Generally, electrons and holes can accumulate on the QDs. Trapping more electrons than holes leads to an increase of the amount of localized electrons. Discharging of the QDs occurs when more holes than electrons are trapped, since the unpaired holes can recombine with localized electrons. The total number of photo-generated electrons and holes that populate or depopulate the QDs depends on their drift and diffusion according to the band profiles. For simplicity, effective rates for QD charging (additional accumulated electrons) and QD discharging (additional accumulated holes) $\Gamma_{\mathrm{c}}$ and $\Gamma_{\mathrm{d}}$, respectively, are introduced. The drift of photo-generated holes and electrons depends sensitively on the applied bias voltage, which enables optical charging for $V=-1.8 \mathrm{~V}$ in Fig. 1(c) and optical discharging for $V=0.0 \mathrm{~V}$ in Fig. 1(d).

For a bias voltage of -1.8 V, electrons created on the surface side of the QDs drift to the QDs and holes drift to the surface (see Fig. 1(e)). A fraction of electrons and holes created on the 2-DEG side also accumulate on the QDs, where they may increase or decrease the number of electrons, respectively. Since the QDs become charged for a bias voltage of $-1.8 \mathrm{~V}$, the amount of electrons that drift to the QDs outnumbers the amount of holes and thus we have $\Gamma_{\mathrm{c}}>\Gamma_{\mathrm{d}}$. For zero bias voltage, holes created on the 2- 
DEG side can drift towards the QDs due to the internal built-in electric field, while the corresponding electrons drift towards the 2-DEG (see Fig. 1(f)). Hence we have $\Gamma_{\mathrm{c}}<\Gamma_{\mathrm{d}}$ and the QDs become optically discharged by the recombination of photo-generated holes with localized electrons. Thus reducing the bias voltage allows to tune the ratio $\Gamma_{\mathrm{d}} / \Gamma_{\mathrm{c}}$ from greater than one to smaller than one.

To investigate the voltage-dependency of the rates in more detail, the bias voltage is changed during illumination with light pulses with widths of $10 \mu \mathrm{s}$ and powers of $730 \mathrm{nW}$ (see Fig. 2(a)). Initially the QDs are discharged and the conductance is large. For a voltage of $-0.6 \mathrm{~V}$ the conductance remains unaltered up to 800 pulses. Smaller voltages lead to a decreasing conductance with voltage-dependent saturation values after 800 pulses (see Fig. 2(b)). Thus the charging of the QDs with electrons can be controlled by tuning the rates $\Gamma_{\mathrm{d}}$ and $\Gamma_{\mathrm{c}}$ with the applied bias voltage. Reducing the bias voltage lowers the ratio $\Gamma_{\mathrm{d}} / \Gamma_{\mathrm{c}}$ and hence electron accumulation on the QDs becomes more efficient.

The conductance of the memristor can be raised or lowered by interband absorption depending on the bias voltage. To possibly realize bi-directional and wavelength-dependent conductance control without changing the electrical input signal, intraband absorption processes are investigated. Current-voltagecharacteristics under cw illumination with infrared light of photon energies 1.32 and $0.95 \mathrm{eV}$ are depicted in Fig. 3(a). Under cw illumination, the width of the low conductance state around zero bias and the area of the pinched hysteresis loop are reduced, which implies smaller amounts of localized electrons. ${ }^{30}$ The photon energies prevent interband absorption of single photons. Because of the low absorption coefficient for intraband absorption, optical powers of 39 and $1.4 \mu \mathrm{W}$ are used for photon energies of 1.32 and $0.95 \mathrm{eV}$, respectively. The conductance for pulsed optical excitation with photon energy $1.32 \mathrm{eV}$, pulse width $100 \mu \mathrm{s}$ and power $3.6 \mathrm{~mW}$ is depicted in Fig. 3(b). The bias voltage during the application of the pulses is varied from -1.0 to $-2.0 \mathrm{~V}$. For large values of the voltage, the increasing conductance saturates 
at enhanced values. The conductance increase is explained by QD-discharge via intraband absorption. ${ }^{32}$ Localized electrons can be excited above the quantum dot surrounding barriers by absorbing a photon as illustrated in the inset of Fig. 3(a). The different saturation values after the application of 1000 pulses are explained by voltage-dependent efficiencies to discharge the QDs via intraband absorption. For small bias voltages, it is more likely to trap the excited electron on the QD again. Consequently, larger applied voltages require less pulses to raise the conductance and in addition lead to enhanced values after the application of 1000 pulses.

Illumination of the presented device with red and infrared light allows to raise and lower the conductance by interband absorption and to raise the conductance by intraband absorption, respectively. The different absorption processes allow to identify a working point, where the QDs can be charged or discharged by optical excitation with different wavelengths. Fig. 4(a) illustrates the conductance versus pulse number for alternating optical excitation with the red and infrared (photon energy: $1.32 \mathrm{eV}$ ) LED. 20 infrared pulses (light power: $2.2 \mathrm{~mW}$ ) followed by 10 red pulses (light power: $1 \mathrm{nW}$ ) are applied in an alternating order. The width of the pulses is $250 \mathrm{~ms}$. The bias voltage during illumination is $-1.6,-1.7$ and $-1.8 \mathrm{~V}$. After every optical pulse a voltage pulse with amplitude $1.0 \mathrm{~V}$ is applied to determine the conductance. For illumination with infrared and red light, the conductance successively increases and decreases, respectively. Thus the conductance state of the memristor can be tuned solely by illumination with light of different wavelengths. Decreasing the bias voltage during illumination lead to lower minimum and maximum values of the conductance modulation, which is explained by a reduced rate $\Gamma_{\mathrm{d}}$ and enhanced $\Gamma_{\mathrm{c}}$.

To model the interband and intraband absorption processes, we use the rate equation

$$
\frac{d n}{d t}=P \cdot\left[H\left(\hbar \omega-E_{g}\right) \Gamma_{c}-n \Gamma_{d}\right]
$$


where $n$ is the number of localized electrons, $P$ the photon incidence rate (proportional to the incident power), $\hbar \omega$ the incident photon energy and $H(\ldots)$ the step function. Charging the QDs by interband absorption is only possible if the photon energy exceeds the effective band gap $E_{\mathrm{g}}$ of GaAs (first term in Eq. (1)). Discharging the QDs by intraband absorption is evident for red and infrared light (second term in Eq. (1)).

Modelling the device performance within the Landauer-Büttiker formalism, the conductance can be reduced to

$$
G(n, \mu)=\frac{\frac{e^{2}}{2 \pi \hbar}\left(\exp \left[\frac{E_{i}(n)-\mu}{k T}\right]+1\right)^{-1}}{1+R \frac{e^{2}}{2 \pi \hbar}\left(\exp \left[\frac{E_{i}(n)-\mu}{k T}\right]+1\right)^{-1}}
$$

$k$ is the Boltzmann constant, $R$ the resistance in series to the wire, $T$ the temperature, $e$ the elementary charge, $\mu$ the electrochemical potential and $E_{i}(n)=E_{i}^{0}+\gamma n+\eta V$ is the transverse subband energy that depends on the QD charge and the applied voltage, $V$, through the efficiencies $\gamma$ and $\eta$. According to Eq. (1), under a train of $N$ pulses with width $\Delta t$ and $\hbar \omega \geq E_{g}$, the localized charge evolves as $n(N)=$ $\frac{\Gamma_{\mathrm{c}}}{\Gamma_{\mathrm{d}}}-\left(\frac{\Gamma_{\mathrm{c}}}{\Gamma_{\mathrm{d}}}-n_{0}\right) e^{-N P \Gamma_{\mathrm{d}} \Delta t}$ with $n_{0}$ being the initial QD charge. Hence, the QDs can be charged or discharged for $n_{0}<\frac{\Gamma_{\mathrm{c}}}{\Gamma_{\mathrm{d}}}$ or $n_{0}>\frac{\Gamma_{\mathrm{c}}}{\Gamma_{\mathrm{d}}}$, corresponding to the conductance decrease or increase displayed in Figs. 1(c) and (d), respectively. In agreement with the experimental data, the limiting value for large $N$ is given by $\frac{\Gamma_{\mathrm{c}}}{\Gamma_{\mathrm{d}}}$ and consequently depends on the bias voltage, as confirmed in Fig. 2(b).

Figs. 4(b) and (c) show the theoretical conductance according to Eqs. (1) and (2) for the same pulse sequence as in the experiment in Fig. 4(a). Similar to the experimental results, the model indicates that the conductance can be successively raised or lowered by varying the photon energy. In addition, the 
amplitude of the conductance modulation is affected by the ratio $\frac{\Gamma_{\mathrm{c}}}{\Gamma_{\mathrm{d}}}$. Applying a periodic sequence of $N_{1}$ pulses with $\hbar \omega>E_{g}$ followed by $N_{2}$ pulses with $\hbar \omega<E_{g}$, the system response becomes stable independent of the initial condition. Combining Eqs. (1) and (2) with the pulse sequence of the experiment, a periodic modulation of the conductance is observed (see Figs. 4(b) and (c)). The maximum and minimum conductances correspond to minimum amounts of localized charge

$$
n_{\min }=\frac{\Gamma_{c}}{\Gamma_{d}} \frac{e^{-N_{1} P \Delta t \Gamma_{d}\left(1-e^{-N_{2} P \Delta t \Gamma_{d}}\right)}}{1-e^{-\left(N_{1}+N_{2}\right) P \Delta t \Gamma_{d}}}
$$

and maximum amounts of localized charge

$$
n_{\max }=\frac{\Gamma_{c}}{\Gamma_{d}} \frac{1-e^{-N_{2} P \Delta t \Gamma_{d}}}{1-e^{-\left(N_{1}+N_{2}\right) P \Delta t \Gamma_{d}}}
$$

respectively. Thus, the ratio $\frac{\Gamma_{\mathrm{c}}}{\Gamma_{\mathrm{d}}}$ determines the amplitude of the conductance modulation, as observed in the experiment (see Fig. 4(a)). Note that by decreasing $\Gamma_{\mathrm{d}}$ in Fig. 4(b) or increasing $\Gamma_{\mathrm{c}}$ in Fig. 4(c), both the amplitude and the lowest value of the conductance oscillation shrinks as observed for more negative bias voltages in Fig. 4(a). Thus we conclude that reducing the bias voltage in the experiment leads to larger rates $\Gamma_{\mathrm{c}}$ and lower $\Gamma_{\mathrm{d}}$.

In summary, we present an electro-photo-sensitive memristor that allows incremental and bi-directional conductance change solely by optical excitation. Within a critical voltage range, illumination with different wavelengths leads to increasing and decreasing conductances that are explained by intraband and interband absorption, respectively. The optical memristance control may pave the way to the realization of artificial synapses for sensory applications. Depending on the wavelength of incoming light, the conductance is raised or lowered. Thus converting information of the light into an electrical signal by sensory neurons is not required and learning can directly be induced by light. 


\section{Acknowledgements:}

The authors gratefully acknowledge financial support from the European Union (FPVII (2007-2013) under grant agreement $\mathrm{n}^{\circ} 318287$ Landauer) as well as the state of Bavaria. The Brazilian authors acknowledge the support of CNPq. V. L.-R. acknowledges the support of FAPESP (grants 2014/02112-3 and 2015/10765-0). 


\section{Figure captions:}

FIG 1. (a) Sketch of the device and circuit diagram. (b) $I$ - $V$-curves in the dark and under cw illumination with a LED emitting photons with energy of $2 \mathrm{eV}$. The arrows indicate the sweep cycle directions. (c) Conductance versus pulse number for pulsed optical excitation with photon energies of $2 \mathrm{eV}$, pulse widths of $10 \mu \mathrm{s}$, and different light powers. The bias voltage during illumination is $-1.8 \mathrm{~V}$. (d) Conductance versus pulse number for pulsed optical excitation with photon energies of $2 \mathrm{eV}$, powers of $44 \mu \mathrm{W}$, and different pulse widths. No bias voltage is applied during illumination. (e),(f) Sketch of the conduction $(\mathrm{CB})$ and valence $(\mathrm{VB})$ band profiles and the electron-hole dynamics for bias voltages of $-1.8 \mathrm{~V}(\mathrm{e})$ and $0 \mathrm{~V}(\mathrm{f})$.

FIG 2. (a) Conductance versus pulse number for pulsed optical excitation at different bias voltages during illumination with photon energies of $2 \mathrm{eV}$. The width and power of the pulses are $10 \mu \mathrm{s}$ and $730 \mathrm{nW}$, respectively. The applied voltage during illumination allows to control the conductance change and the saturation conductance value after 800 pulses. (b) Conductance after 800 pulses versus bias voltage. The data is determined from similar curves as in (a). Lower voltages lead to lower values of the conductance.

FIG 3. (a) Current-voltage-characteristics in the dark and under cw illumination with photon energies of 1.32 and $0.95 \mathrm{eV}$. Under illumination, the area of the pinched hysteresis loop is reduced corresponding to a QD discharging. The inset illustrates the band profiles and the discharging of the QD via intraband absorption. (b) Conductance for pulsed optical excitation with photon energy $1.32 \mathrm{eV}$ for pulse widths of $100 \mu \mathrm{s}$ and power of $3.6 \mathrm{~mW}$. Tuning the bias voltage during illumination allows to control the conductance change and hence the discharging of the QDs via intraband absorption.

FIG 4. (a) Conductance for pulsed optical excitation with the red (photon energy: $2.0 \mathrm{eV}$ ) and infrared LED (photon energy: $1.32 \mathrm{eV}$ ), measured for different bias voltages during illumination. $G$ is successively enhanced and lowered by the infrared and red light pulses, respectively. Lower voltages lead to reduced

minimum and maximum values of the conductance modulation. (b) Theoretically obtained conductances for the conditions used in the experiment in panel (a) for constant $\Gamma_{\mathrm{c}}$ and varying $\Gamma_{\mathrm{d}}$. For the squares, circles and triangles, $P \Gamma_{\mathrm{d}} \Delta t$ is $0.15,0.15 / 2$ and $0.15 / 3$, respectively. Lower $\Gamma_{\mathrm{d}}$ lead to smaller minimum and maximum conductance values. (c) Similar simulations as in (b), but for constant $\Gamma_{\mathrm{d}}$, varying $\Gamma_{\mathrm{c}}$ and $P \Gamma_{\mathrm{d}} \Delta t=0.15$. 


\section{References:}

${ }^{1}$ N. Kirstaedter, N. N. Ledentsov, M. Grundmann, D. Bimberg, V. M. Ustinov, S. S. Ruvimov, M. V. Maximov, P. S. Kop'ev, Z. I. Alferov, U. Richter, P. Werner, U. Gösele, and J. Heydenreich, Electronics Letters 30, 1416 (1994).

${ }^{2}$ Z. Yuan, B. E. Kardynal, R. M. Stevenson, A. J. Shields, C. J. Lobo, K. Cooper, N. S. Beattie, D. A. Ritchie, and M. Pepper, Science 295, 102 (2002).

${ }^{3}$ M. Kroutvar, Y. Ducommun, D. Heiss, M. Bichler, D. Schuh, G. Abstreiter, and J. J. Finley, Nature 432, 81 (2004).

${ }^{4}$ A. Marent, T. Nowozin, M. Geller, and D. Bimberg, Semicond. Sci. Technol. 26, 014026 (2011).

${ }^{5}$ P. Pavan, R. Bez, P. Olivo, and E. Zanoni, Proceedings of the IEEE 85, 1248 (1997).

${ }^{6}$ L. Guo, E. Leobandung, and S. Y. Chou, Science 275, 649 (1997).

${ }^{7}$ J. J. Finley, M. Skalitz, M. Arzberger, A. Zrenner, G. Böhm, and G. Abstreiter, Appl. Phys. Lett. 73, 2618 (1998).

${ }^{8}$ H. Pettersson, L. Baath, N. Carlsson, W. Seifert, and L. Samuelson, Appl. Phys. Lett. 79, 78 (2001).

${ }^{9}$ M. Kroutvar, Y. Ducommun, J. J. Finley, M. Bichler, G. Abstreiter, and A. Zrenner, Appl. Phys. Lett. 83, 443 (2003).

${ }^{10}$ M. A. Rowe, E. J. Gansen, M. Greene, R. H. Hadfield, T. E. Harvey, M. Y. Su, S. W. Nam, R. P. Mirin, and D. Rosenberg, Appl. Phys. Lett. 89, 253505 (2006).

${ }^{11}$ G. Yusa and H. Sakaki, Appl. Phys. Lett. 70, 345 (1997).

${ }^{12}$ X. Yang, X. Xu, X. Wang, H. Ni, Q. Han, Z. Niu, and D. A. Williams, Appl. Phys. Lett. 96, 083503 (2010).

${ }^{13}$ M. Ziegler, M. Oberländer, D. Schroeder, W. H. Krautschneider, and H. Kohlstedt, Appl. Phys. Lett. 101, 263504 (2012).

${ }^{14}$ I. Orak, M. Ürel, G. Bakan, and A. Dana, Appl. Phys. Lett. 106, 233506 (2015).

${ }^{15}$ D. B. Strukov, G. S. Snider, D. R. Stewart, and R. S. Williams, Nature 453, 80 (2008).

${ }^{16}$ L. O. Chua. IEEE Trans. Circuit Theory 18, 507 (1971).

${ }^{17}$ M. Di Ventra and Y. V. Pershin, Nat. Physics 9, 200 (2013).

${ }^{18}$ A. Emboras, I. Goykhman, B. Desiatov, N. Mazurski, L. Stern, J. Shappir, and U. Levy, Nano Lett. 13, 6151 (2013).

${ }^{19}$ C. He, J. Li, X. Wu, P. Chen, J. Zhao, K. Yin, M. Cheng, W. Yang, G. Xie, D. Wang, D. Liu, R. Yang, D. Shi, Z. Li, L. Sun, and G. Zhang. Adv. Mat. 25, 5593 (2013).

${ }^{20}$ A. A. Zakhidov, B. Jung, J. D. Slinker, H. D. Abruña, and G. G. Malliaras, Organic Electronics 11, 150 (2010).

${ }^{21}$ M. Ungureanu, R. Zazpe, F. Golmar, P. Stoliar, R. Llopis, F. Casanova, and L. E. Hueso, Adv. Mat. 24, 2496 (2012).

22 J. Park, S. Lee, J. Lee, and K. Yong, Adv. Mat. 25, 6423 (2013).

${ }^{23}$ B. Sun, W. Zhao, L. Wei, H. Li, and P. Chen. Chem. Commun. 50, 13142 (2014).

${ }^{24}$ J. Park, S. Lee, and K. Yong, Nanotechnology 23, 385707 (2012).

${ }^{25}$ H. Tan, G. Liu, X. Zhu, H. Yang, B. Chen, X. Chen, J. Shang, W. D. Lu, Y. Wu, and R.-W. Li, Adv. Mat. 27, 2797 (2015).

${ }^{26}$ A. Bera, H. Peng, J. Lourembam, Y. Shen, X. W. Sun, and T. Wu, Adv. Funct. Mat. 23, 4977 (2013). 
${ }^{27}$ C. D. Wright, Y. Liu, K. I. Kohary, M. M. Aziz, and R. J. Hicken, Adv. Mat. 23, 3408 (2011).

${ }^{28}$ B. Gholipour, P. Bastock, C. Craig, K. Khan, D. Hewak, and C. Soci, Adv. Optical Mat. 3, 635 (2015).

${ }^{29}$ C. Schneider, A. Huggenberger, T. Sünner, T. Heindel, M. Strauß, S. Göpfert, P. Weinmann, S. Reitzenstein, L. Worschech, M. Kamp, S. Höfling, and A. Forchel, Nanotechnology 20, 434012 (2009).

30 P. Maier, F. Hartmann, T. Mauder, M. Emmerling, C. Schneider, M. Kamp, S. Höfling, and L. Worschech, Appl. Phys. Lett. 106, 203501 (2015).

${ }^{31}$ L. O. Chua, Semicond. Sci. Technol. 29, 104001 (2014).

32 T. Nozawa, H. Takagi, K. Watanabe, and Y. Arakawa, Nano Lett. 15, 4483 (2015). 
Figure 1:
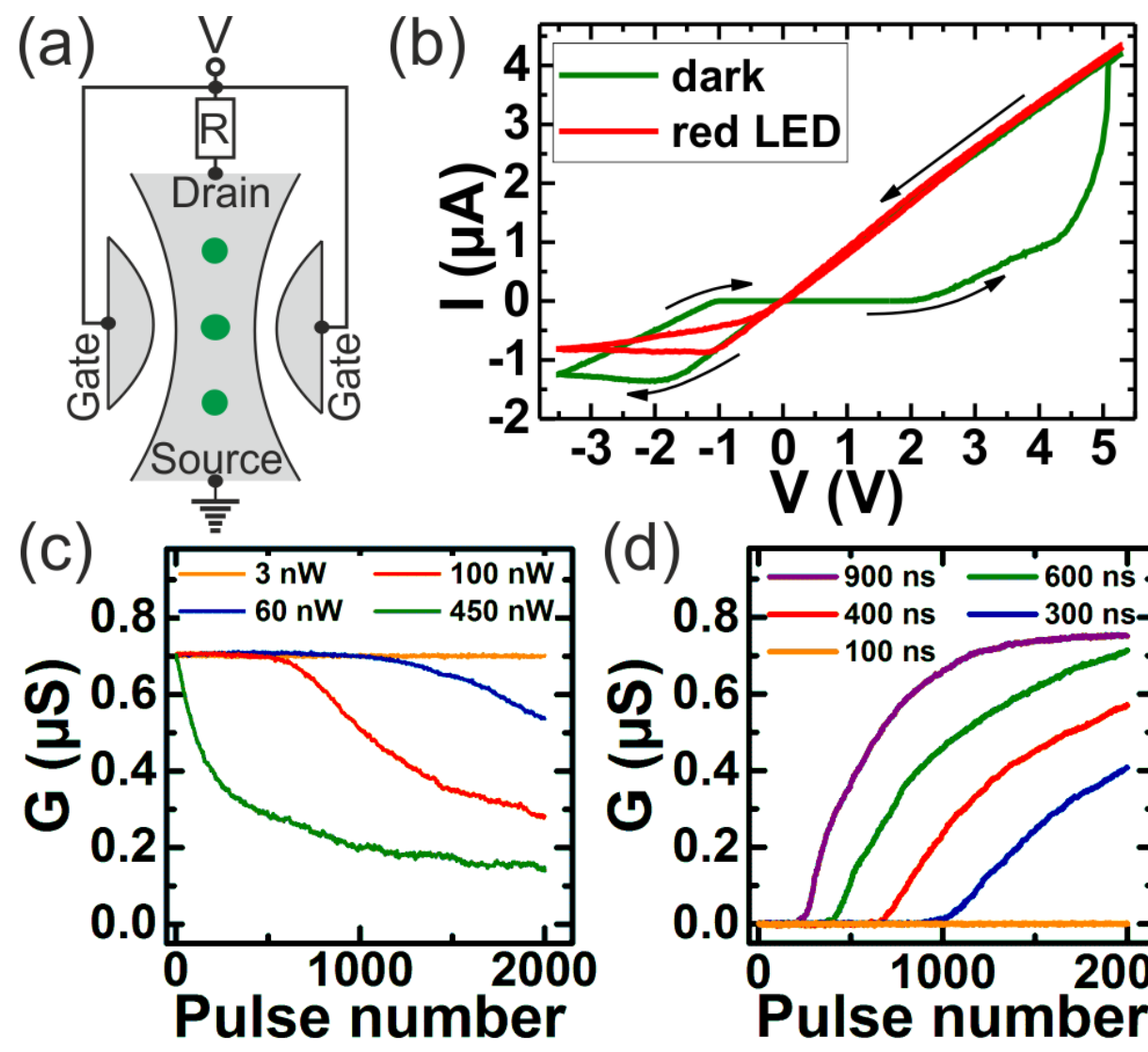

(e)
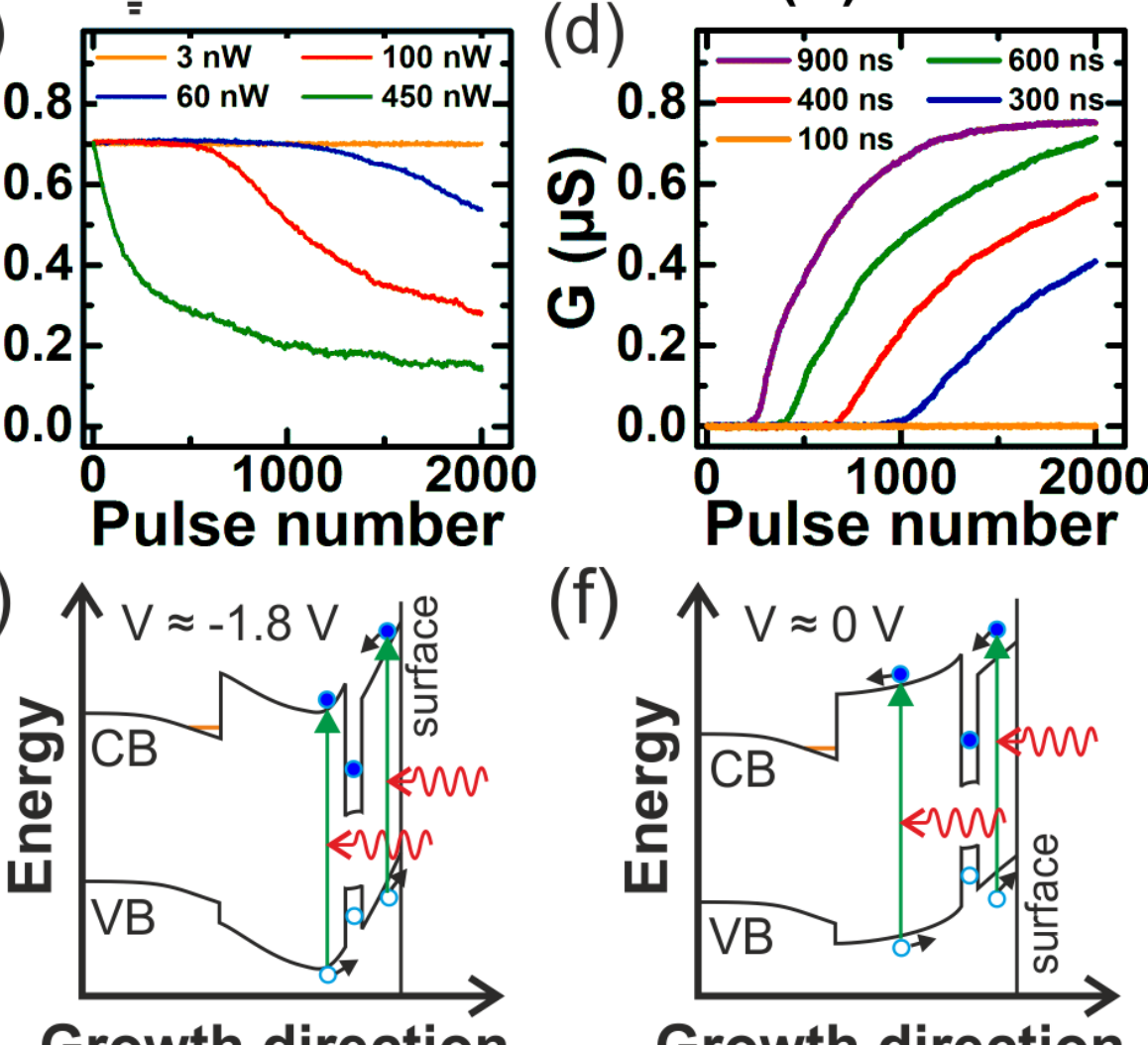

Growth direction

(f)

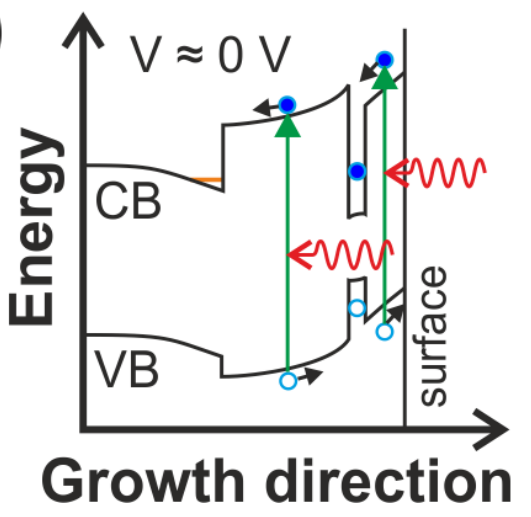


Figure 2:
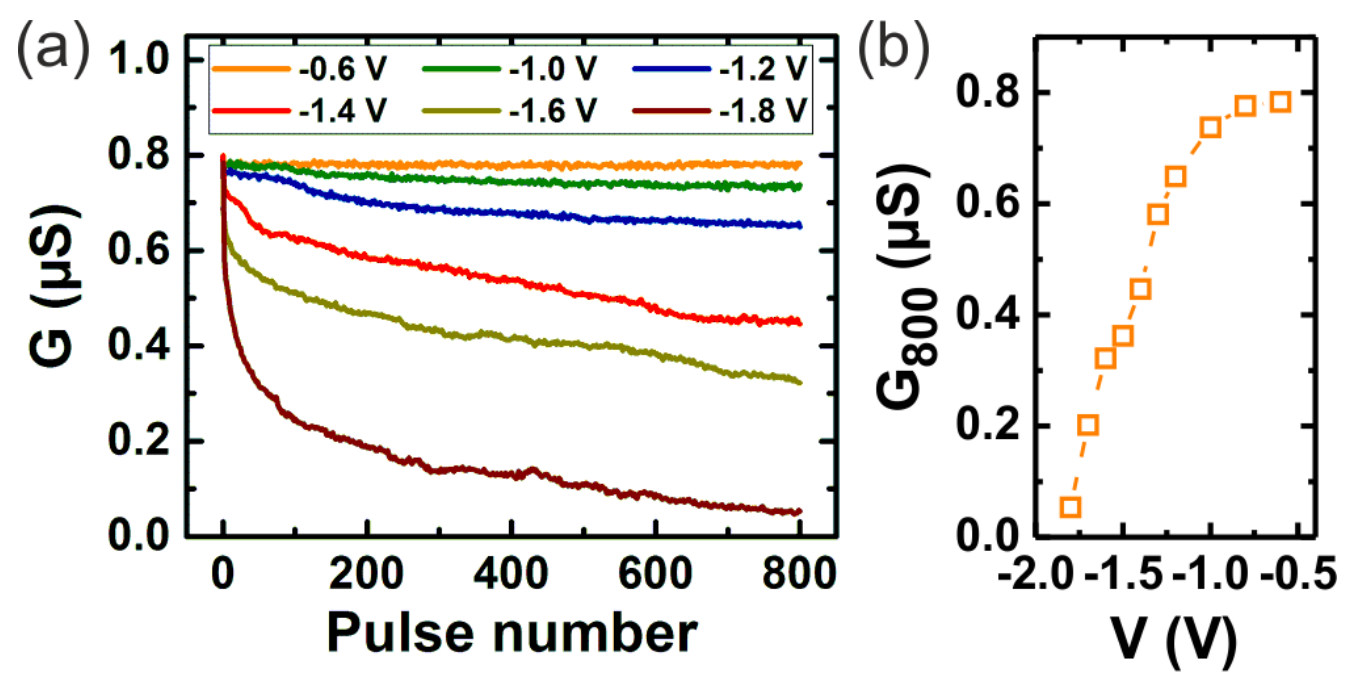
Figure 3:
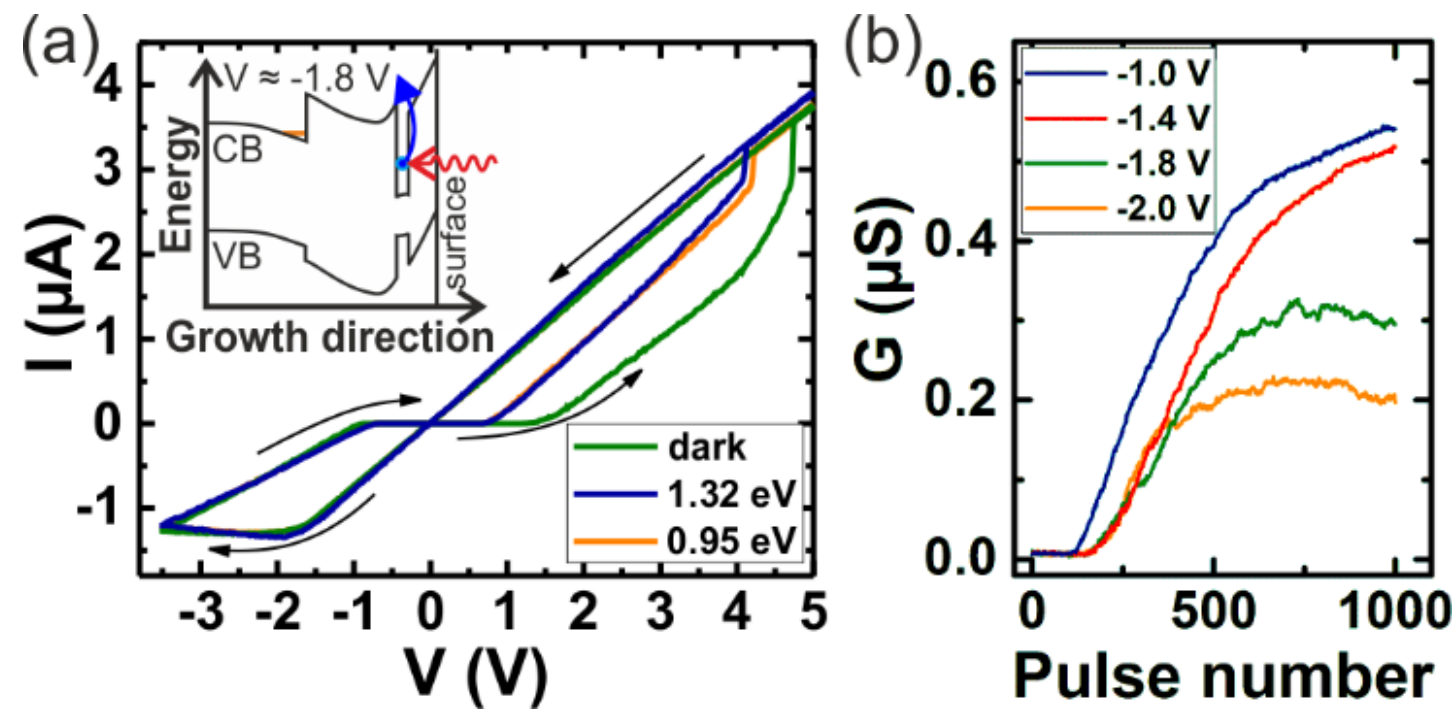
Figure 4:

(a) infrared LED red LED

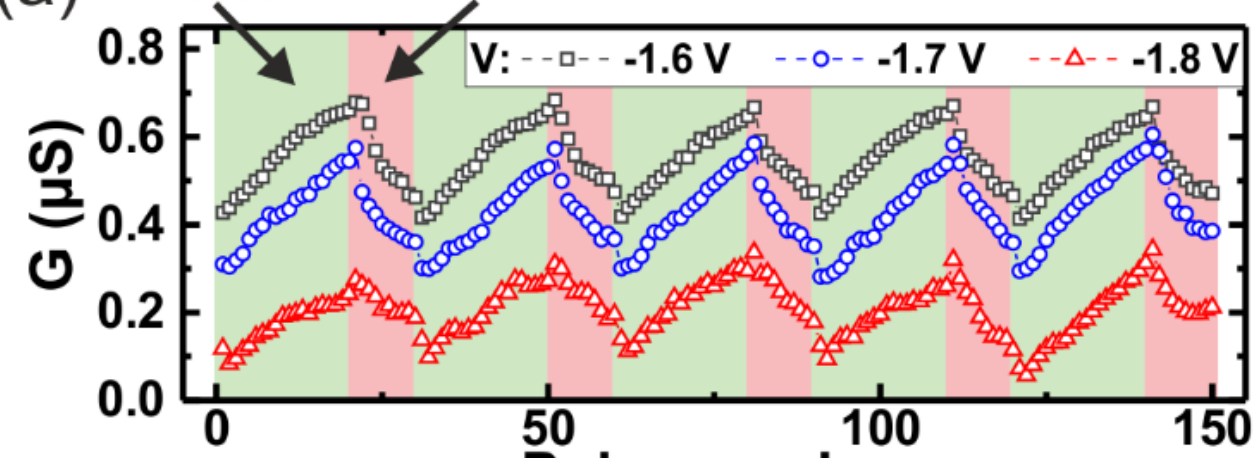

(b)

Pulse number
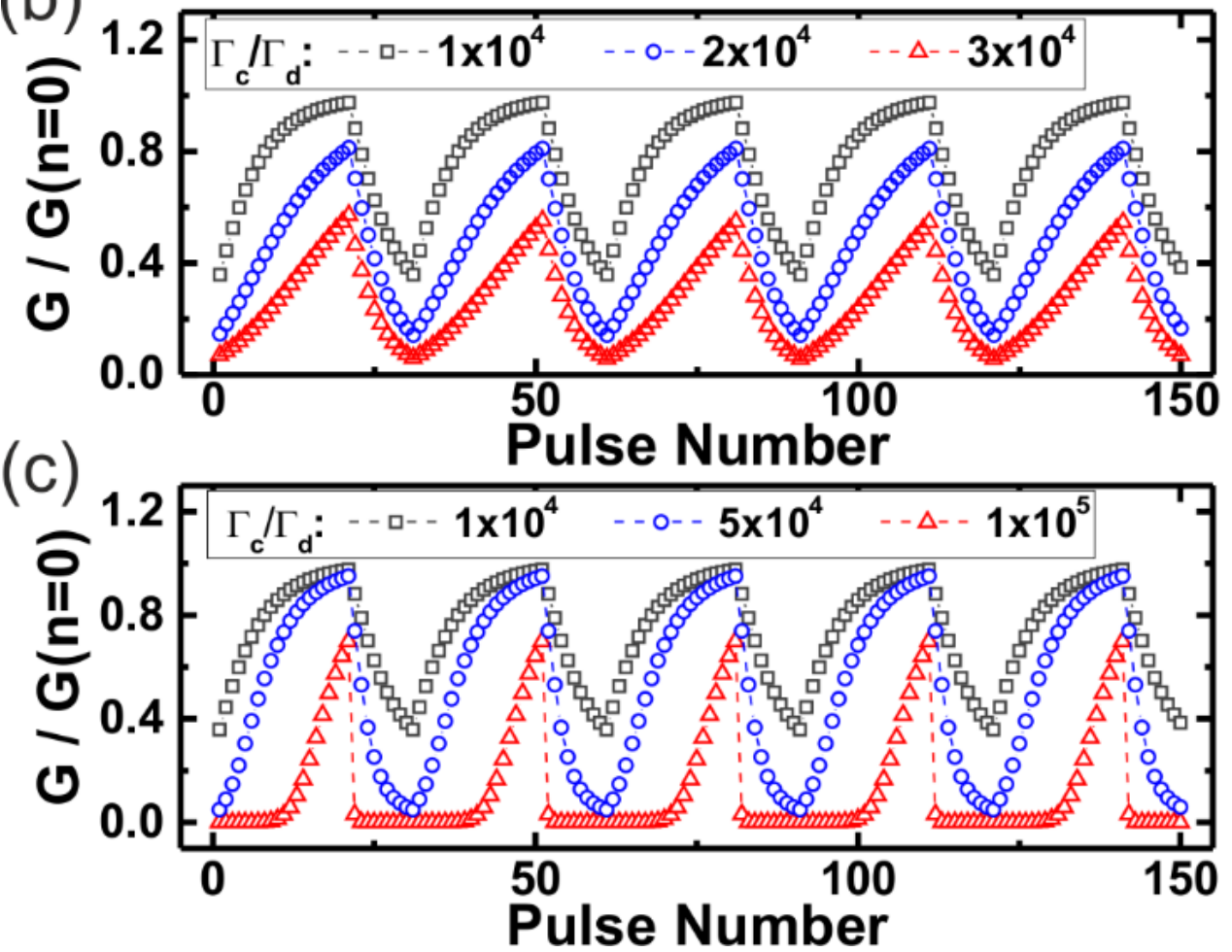